EMJ

\title{
Improving post-hypoglycaemic patient safety in the prehospital environment: a systematic review
}

D Fitzpatrick and E A S Duncan

Emerg. Med. J. 2009;26;472-478

doi:10.1136/emj.2008.062240

Updated information and services can be found at:

http://emj.bmj.com/cgi/content/full/26/7/472

\section{These include:}

References This article cites 18 articles, 7 of which can be accessed free at: http://emj.bmj.com/cgi/content/full/26/7/472\#BIBL

Rapid responses $\quad \begin{aligned} & \text { You can respond to this article at: } \\ & \text { http://emj.bmj.com/cgi/eletter-submit/26/7/472 }\end{aligned}$

Email alerting Receive free email alerts when new articles cite this article - sign up in the box at service the top right corner of the article

Topic collections Articles on similar topics can be found in the following collections

Patients (1886 articles)

Diabetes (3362 articles)

Notes

To order reprints of this article go to:

http://journals.bmj.com/cgi/reprintform

To subscribe to Emergency Medicine Journal go to:

http://journals.bmj.com/subscriptions/ 


\title{
Improving post-hypoglycaemic patient safety in the prehospital environment: a systematic review
}

\author{
D Fitzpatrick, E A S Duncan
}

NMAHP Research Unit, The University of Stirling, Stirling, UK

Correspondence to: Mr D Fitzpatrick, Scottish Ambulance Service NMAHP Research Unit, Iris Murdoch Building, The University of Stirling, Stirling FK9 4LA,

Scotland, UK; david.fitzpatrick@ stir.ac.uk

Accepted 1 November 2008

\section{ABSTRACT}

To determine the extent to which post-hypoglycaemic patients with diabetes, who are prescribed oral hypoglycaemic agents $(\mathrm{OHA})$ are at risk of repeat hypoglycaemic events (RHE) after being treated in the prehospital environment and whether they should be transported to hospital regardless of their post-treatment response, a systematic literature review was carried out using an overlapping retrieval strategy that included both published and unpublished literature. Retrieved papers were reviewed by each author for inclusion. Disagreements regarding inclusion were resolved through discussion. Ninety-eight papers and other relevant material were retrieved using the developed search strategy. Twentythree papers and other relevant material were included in the final review. A narrative synthesis of the findings is presented. Although several case reports demonstrate the risks associated with repeat or prolonged hypoglycaemia, the review was unable to locate any specific high quality research in this area. Consequently, caution is required in interpreting the findings of the studies. Post-hypoglycaemic patients treated in the prehospital environment have a $2-7 \%$ risk of experiencing a RHE within $48 \mathrm{~h}$. The literature retrieved in this study recognises the potential for OHA to cause RHE. However, the extent to which this occurs in practice remains unknown. This lack of evidence has led to the recommendation that conservative management, through admission to hospital, is appropriate. The review concludes with recommendations for both practice and research.

Diabetes is a growing health concern placing increasing pressure on NHS resources. ${ }^{1}$ In the UK 2.3 million people are currently diagnosed with the condition; a figure predicted to rise to 3 million by the end of the decade. ${ }^{2}$ Audit data from one of the UK's largest ambulance services indicate that approximately $50 \%$ of the 6500 diabetes-related emergency calls per annum were for patients with hypoglycaemia. (R Lawrenson, Scottish Ambulance Service Audit Manager, personal communication, 2008).

In response to continuing increases in emergency department admissions, ${ }^{3}$ UK ambulance services have identified new ways of working aimed at reducing unnecessary transportations and attendances at emergency departments. Initiatives such as "treat and refer" or "see and treat" (S\&T) guidelines have enabled ambulance clinicians to attend, treat, discharge or refer patients with a defined list of conditions as appropriate. ${ }^{4-6}$ One condition typically included in such guidelines is hypoglycaemia in diabetes.

This review focuses on patients with type II diabetes who are prescribed oral hypoglycaemic agents (OHA). OHA are used to treat some patients with type II diabetes when there is either insulin resistance or when the pancreas produces insufficient quantities of insulin to meet the body's requirements. ${ }^{78}$ In the latter, their action stimulates the secretion of stored insulin from pancreatic beta cells, which then acts at a cellular level enabling the uptake and utilisation of glucose by the cell. ${ }^{8}$ Recurrent and prolonged hypoglycaemia resulting from renal insufficiency, drug interaction or accidental/deliberate overdose are known risks of OHA, particularly the sulphonylurea group..$^{7-9}$ In the prehospital setting a post-hypoglycaemic patient may be discharged by the ambulance clinician after apparent recovery, only to have a subsequent event hours or even days later. These risks have resulted in some, ${ }^{510-13}$ but not all, ${ }^{414}$ ambulance services advising transportation to hospital for all post-hypoglycaemic patients with type II diabetes treated with OHA, regardless of treatment response. Such service variation highlights the inconsistencies in ambulance service hypoglycaemia guidelines both in the UK and internationally. ${ }^{4}$ 10-14

\section{AIM}

To determine the extent to which post-hypoglycaemic patients with diabetes who are prescribed OHA are at risk of repeat hypoglycaemic events (RHE) after being treated in the prehospital environment, and whether they should be transported to hospital regardless of their post-treatment response.

\section{METHODS}

A systematic literature review was conducted. The following search strategy was used to retrieve relevant material: (1) Medline (using Pubmed) was searched using the following search strategy: ("diabetes"[All Fields] OR "diabetes mellitus"[All Fields] OR "diabetic"[All Fields]) AND (("sulphonylurea"[All Fields] OR "sulfonylurea"[All Fields]) OR "rebound hypoglycaemia"[All Fields] OR "diabetic emergency"[All Fields] OR "oral agents"[All Fields] OR "oral hypoglycaemic agent\$"[All Fields] OR ("hypoglycaemia"[All Fields] OR "hypoglycaemia"[All Fields] OR "hypoglyc\$"[All Fields]) OR "glucose"[All Fields] OR "dextrose"[All Fields] OR "glucagon"[All Fields]) AND ("pre-hospital"[All Fields] OR "prehospital"[All Fields] OR "ambulance"[All Fields] OR "paramedic"[All Fields] OR ("therapy"[Subheading] OR "therapy"[All Fields] OR "treat"[All Fields]) AND refer[All Fields]) OR "see and treat"[All Fields]). (2) 
Electronic searching of the Cochrane Library and clinicaltrials. gov using adaptations of the above search terms. (3) Hand searching of clinical text books and key journals: BNF pharmacology 2006; Emergency Medicine Journal (January 2000 to March 2007); Joint Royal College Ambulance Liaison Committee National Clinical Guidelines (2006). (4) Grey literature (unpublished material): "Pathfinder" course material. (5) Personal communication with other ambulance services and diabetologists.

\section{Inclusion/exclusion criteria}

Peer reviewed papers, letters and unpublished documents were all included in the review. Retrieved material was excluded if it did not include patients with type II diabetes treated with OHA, the topic was not of direct relevance, or the paper was not published in English.

Titles and abstracts were screened for inclusion by one of the authors (DF). All potentially relevant papers and material were retrieved and the full documents were then read by both authors to check that each met the inclusion criteria. Each paper was then categorised according to publication source and research method (fig 1). References of electronically retrieved but excluded papers are available from the authors on request.

\section{ANALYSIS}

Due to the small number, low quality and data heterogeneity of included quantitative research studies a meta-analysis of the findings was not conducted. Instead a descriptive summary of the included paper's sources and research design was developed and a narrative synthesis of key themes from these papers is provided.

\section{Findings}

Table 1 outlines the sources of literature and types of study design of the included papers. Eighteen papers were retrieved from peer-reviewed journals, one internet site and four publications from other sources were also included. Only one controlled trial was found in this area. ${ }^{12}$ Eight of the included papers were cohort studies. ${ }^{613-20}$ Six papers were literature

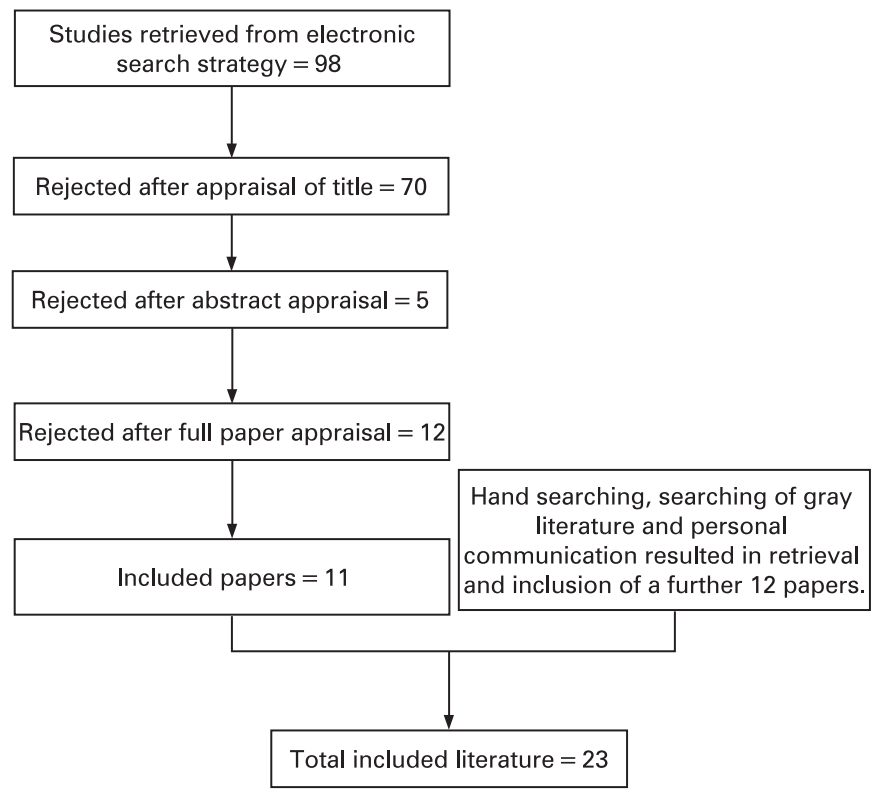

Figure 1 A flow chart of included literature. reviews. ${ }^{821-24}$ Two presented case reports ${ }^{25} 26$ and one reported a retrospective case note analysis study. ${ }^{14}$ In addition, four guidelines $s^{41011}$ and one well recognised reference book ${ }^{7}$ were included.

Of the included studies from the retrieved literature ( $\mathrm{n}=18), 12^{6}{ }^{12-18} 22-25$ included patients who had had hypoglycaemic events and were treated with OHA. Twelve 9 13 15-17 192125 emphasised the inherent dangers of these drugs, and

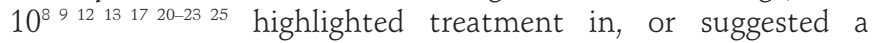
treatment only available within, the hospital environment. Care was taken to ensure no double counting of individual studies occurred.

\section{RESULTS}

\section{Incidence of OHA-induced hypoglycaemia}

Of the included studies, which followed patients between 6 months and 3 years, the incidence of OHA-induced hypoglycaemia (in type II diabetes) was predominantly reported as being between $0.8 \%$ and $12 \%{ }^{6}{ }^{151621}$ However, one study reported an incidence of $23 \%$ over a 3 -year period. ${ }^{13}$

\section{Risks and causes of OHA-induced hypoglycaemia}

The potential for hypoglycaemia was highlighted as the most significant risk associated with OHA. ${ }^{724}$ The primary risk to any hypoglycaemic patient is reduced levels of consciousness, leading to airway compromise, seizure, cerebral oedema (potentially resulting in permanent neurological deficit) and in rare cases, death. ${ }^{84}$ Other risks such as prolonged hypoglycaemia or RHE result from the long-acting nature of some OHA, particularly the sulphonylurea group. ${ }^{8132425}$ Cases have been described in which hypoglycaemic patients prescribed OHA have been treated appropriately with intravenous glucose only to experience RHE hours or even days after the initial event. ${ }^{17} 25$

Two reviews ${ }^{82}$ describe the general risk factors associated with hypoglycaemia in patients treated with OHA. These include the elderly, those with a history of vascular disease, renal failure, reduced food intake, alcohol consumption and drug interactions. Specific examples of these risk factors in the included papers include medication interactions, ${ }^{826}$ quiescent renal or hepatic disease $e^{25}$ and accidental or deliberate ingestion. ${ }^{8}$ Many of these arise from the clinicians' failure to consider the contribution of such risk factors to either an initial or RHE and illustrate how ignoring such risk factors unnecessarily exposes the patient to the dangers associated with hypoglycaemia.

\section{Repeat calls for hypoglycaemic events}

Five of the papers included in this review directly discussed repeat emergency calls for hypoglycaemic events. ${ }^{14}{ }^{16-19}$ These papers were examined to identify the rates of RHE and whether OHA were viewed as a contributory factor. There was no standard definition of "recurrence" in the literature. Reported recurrence times ranged between 24 and $120 \mathrm{~h}$, with the incidence of RHE within a 48 -h period varying between $2 \%$ and 7\%. ${ }^{14-19}$ No studies were detailed enough to determine how many of these RHE were directly related to OHA.

\section{Transport to hospital}

Roberts and $\mathrm{Smith}^{23}$ identified post-hypoglycaemic patients treated with OHA as high risk and recommended that they be transported to hospital; no rationale was provided. Brackenridge et $a l^{20}$ emphasise that patients on OHA are "special cases" ( $p$ 185 ) and suggest the "threshold for admission" ( $p$ 185) to be 
Table 1 Study design and core findings of included papers

\begin{tabular}{lllll}
\hline $\begin{array}{l}\text { Author, date and } \\
\text { country }\end{array}$ & Patient group & Study design & Core findings and outcomes & Limitations/weaknesses \\
\hline
\end{tabular}

\begin{tabular}{|c|c|c|c|c|}
\hline $\begin{array}{l}\text { Mattila et al, }{ }^{12} 2004, \\
\text { Finland }\end{array}$ & $\begin{array}{l}\text { Investigation of the clinical epidemiology } \\
\text { of severe out-of-hospital hypoglycaemia }\end{array}$ & Control trial & $90 \%(n=62)$ of patients were left at scene & $\begin{array}{l}\text { Only } 8.5 \% \text { ( } n=69 \text { ) of patients left at } \\
\text { home were included in this study }\end{array}$ \\
\hline
\end{tabular}

Finland of severe out-of-hospital hypoglycaemia

11 months (Feb-Dec 2001)

69 Participants

Steinmetz et $a l_{{ }^{18}}^{18} 2006$, Assessed patients preferences to locality Prospective Denmark of treatment, ie, hospital or home. cohort study 139 Participants
Prospective cohort study pathway to a DSN 3 Months (Dec 2002-March 2003) 38 Participants
Brackenridge et al, 2006, UK
Exploration of the use of emergency services by people with diabetes 12-Month audit (Oct 2000-Sept 2001) 89 Participants
Cain et $a l_{{ }^{17}}{ }^{17} 2003$, Canada Determined the outcomes of patients treated and not transported for hypoglycaemia and identified criteria for those who do not require to be transported

10 Months (Aug-June 2001)

220 Participants
$55 \%$ ( $n=22$ ) of questionnaire respondents had a RHE within 3-month follow-up period, none within $24 \mathrm{~h}$

The practice of leaving selected patients at the scene after assessment and treatment was found to be effective, safe and economical All patients with type 2 diabetes prescribed $\mathrm{OHA}$ were transported to hospital $6 \%(n=8)$ patients treated and discharged at This intervention was limited to the scene were admitted to hospital within $48 \mathrm{~h}$ physicians

Involvement of $\mathrm{OHA}$ is unknown

$53 \%(\mathrm{n}=20)$ of referred patients had medication altered, $37 \%(n=14)$ required ongoing review. Of participants who returned questionnaire, $73 \%(\mathrm{n}=19)$ felt more able to treat a hypoglycaemic episode in the future.

Recommends specialist pathways to DSN for patients who have had a hypoglycaemic event

Highlights the need for medication alteration and expert advice for patients

Prospective cohort study

Management of hypoglycaemia by the emergency services was varied and not always appropriate

$11 \%(\mathrm{n}=10)$ of sample were admitted to hospital

Many patients who attend ED were discharged with no follow-up arrangements. Education is required for healthcare professionals. Highlights challenges of identifying people who need to be admitted to hospital.

States that patients treated with OHA should be treated as "special cases"

Prospective cohort study

Repeat hypoglycaemic events are reported to be Unable to determine OHA involvement common, however, recurrences within $48 \mathrm{~h}$ are in recurrence of hypoglycaemia not

From the 220 hypoglycaemic emergencies, 145 patients were left at home, $27 \%(n=40)$ of whom made a repeat call within the study period; $2 \%(n=3$ ) of these were within $48 \mathrm{~h}$. From the 75 patients transported to hospital, $22.7 \%(\mathrm{n}=17)$ reported a $\mathrm{RHE}$, with $4 \%$ ( $\mathrm{n}=3$ ) occurring within a 48-h period.

Given the high incidence of repeat hypoglycaemic episodes, paramedics and physicians need to emphasise the importance of follow-up

Holstein, ${ }^{13} 2003$

Obtain reliable data on the care of

Prospective Germany prehospital diabetic emergencies with a cohort study view to improving quality of care 3 Years (Jan 1997-Dec 2000) 213 Patients
Training of emergency teams with a specific intervention improved quality of treatment and prognosis outcome for patients

Treatment of severe hypoglycaemia at the scene Limited number of sulfonylurea-induced was deemed to be safe in patients with type 1 hypoglycaemia, much larger trials diabetes who had previously received structured including comorbidity, type of OHA and patient education dose would be required to substantiate the results of this study

Mortality rates for sulfonylurea-induced

hypoglycaemia dropped from $4.9 \%(n=2)$ to $0 \%$ 
Table 1 Continued

\begin{tabular}{llll}
\hline $\begin{array}{l}\text { Author, date and } \\
\text { country }\end{array}$ & Patient group & Study design & Core findings and outcomes \\
\hline Spiller, ${ }^{9}$ 1998, USA & $\begin{array}{l}\text { Focuses on antidiabetic medications in } \\
\text { overdose (deliberate and accidental) }\end{array}$ & $\begin{array}{l}\text { Systematic } \\
\text { literature review }\end{array}$ & $\begin{array}{l}\text { Overdose with antidiabetic drugs produces } \\
\text { major morbidity, requires intensive care } \\
\text { medicine and prolonged hospital stays } \\
\text { Monitored for at least } 24 \mathrm{~h}\end{array}$
\end{tabular}

Limitations/weaknesses

No search strategy defined, difficulty in reproducing review

Narrative synthesis of findings, not meta-analysed

When overdose does occur prompt recognition results in an improvement in successful outcomes $\begin{array}{lll}\text { Harrigan et } a l^{8}{ }^{8} 2001, & \begin{array}{l}\text { Review of oral antidiabetic agents } \\ \text { describing the treatment of type } 2\end{array} & \text { Review } \\ \text { USA } & \end{array}$ diabetes regarding the pharmacology, toxicity and treatment

Pharmacology of OHA described, adverse effects, ie, hypoglycaemia, drug interactions and in reproducing review toxicities

Patients with hypoglycaemia in the setting of therapeutic error, impaired renal function etc should be admitted for observation

Describes prehospital treatment for diabetesrelated emergencies. Prolonged hypoglycaemia in patients treated with $\mathrm{OHA}$ is highlighted.

Search strategy is not defined, difficulty in reproducing review. Some of the treatments are either specific to region or dated.

Patients on $\mathrm{OHA}$ are at risk of prolonged hypoglycaemia

Hypoglycaemia is a major potential adverse effect of sulfonylurea agents. Causes and treatments are described, specifically the use of octreotide. Clearly describes pharmacological actions of sulfonylureas.

Advises transportation of all posthypoglycaemic patients with diabetes treated with OHA to hospital

Basics, $^{10}$ Pathfinder
guidelines, 2004, UK Guideline

Welsh Ambulance $\mathrm{n} / \mathrm{a}$

Service, ${ }^{5}$ T\&R guidelines, 2006, Wales

Joint Royal College

Ambulance Liaison

Committee, ${ }^{11}$ 2006, UK

Scottish Ambulance

Service, ${ }^{4}$ T\&R guideline,

2004, Scotland

British National

Focuses on the antidotal treatment of
sulfonylurea-induced hypoglycaemia with octreotide

Review

Formulary, ${ }^{7} 2006$, UK
Guideline

Advises transportation to hospital for patients treated with $\mathrm{OHA}$ who have experienced a hypoglycaemic event have experienced a hypoglycaemic event should be transported to hospital

Guideline

Does not specifically advise transportation to hospital for patients treated with OHA who have experienced a hypoglycaemic event

The BNF provides specific detail on sulphonylurea-induced hypoglycaemia and highlights the prolonged effects and the need for treatment in hospital
Guideline Advises that all patients treated with $\mathrm{OHA}$ who n/a

BNF, British Nationial Formulary; DSN, diabetic specialist nurse; ED, emergency department; EMS, emergency medical services; iv, intravenous; OHA, oral hypoglycaemic agent; $\mathrm{RHE}$, repeat hypoglycaemic event; T\&R, treat and refer.

low, while Socransky et a ${ }^{16}$ state that patients taking OHA who have had a hypoglycaemic event should be convinced to travel to hospital. The British National Formulary $(\mathrm{BNF})^{7}$ recommends that such patients should always be treated in hospital. A recent review ${ }^{9}$ supports this stance and recommends patients are observed for at least $24 \mathrm{~h}$; whereas less conservatively, Harrigan et $a l^{8}$ suggest a minimal observation time of $8 \mathrm{~h}$. The length of time patients should be observed, therefore, remains unclear.

The reviews by Murphy and Colwell ${ }^{22}$ and Harrigan et al reveal the dangers of hypoglycaemia caused by OHA and highlight treatments such as continuous glucose infusions and constant blood glucose monitoring. A strong case for such treatments and transportation of patients with OHA-induced hypoglycaemia is demonstrated in the study by Holstein et $a l,{ }^{13}$ in which the authors specifically targeted sulphonylurea-induced hypoglycaemic patients with a treatment protocol. This consisted of mandatory additional glucose infusions and hospital admission for further treatment and observation. The study's pre-intervention statistics on mortality rates associated with all sulphonylurea-induced hypoglycaemia were $4.9 \%$ and $1.7 \%$ for all other causes. After intervention, mortality rates from severe hypoglycaemia caused by sulphonylureas were reduced to $0 \%$.

\section{DISCUSSION}

This systematic review has retrieved and analysed 23 documents in an attempt to establish if post-hypoglycaemic patients with diabetes who are prescribed OHA are at risk of RHE after being treated in the prehospital environment and whether such patients should be transported to hospital regardless of treatment response.

\section{Risks associated with OHA}

Although a relatively rare occurrence, patients with diabetes treated with OHA can and do experience hypoglycaemic episodes. This review clearly exposes the risks associated with OHA and the possible dangers of RHE when leaving posthypoglycaemic patients treated with OHA at home. ${ }^{720232526} \mathrm{~A}$ decision not to transport these patients may result in unnecessary and unacceptable risks, including repeat or prolonged hypoglycaemia leading to seizure, cerebral oedema, permanent neurological deficit and, rarely, death. ${ }^{89}$

\section{Repeat calls and OHA}

It cannot be determined from the five papers ${ }^{14}{ }^{16-19}$ documenting repeat call-outs if OHA were directly involved, but it is evident 
that unrecognised risks, such as those outlined in the study by Harrigan et $a l^{8}$ and failure to treat accordingly may lead to a RHE posing a direct risk to patients. Repeat calls also directly impact on ambulance resources reducing ambulance availability and consequently affecting response times. On the basis of the limited available evidence, the safest action to be taken by ambulance clinicians attending a post-hypoglycaemic patient with diabetes treated with OHA is to transport them to hospital to be admitted for a period of observation, appropriate treatment and follow-up care. The potential benefits of admission may include extended monitoring, continuous glucose infusions, pharmacological interventions, exploration of underlying causes and medication alteration if required. In the UK the standard prehospital treatment for hypoglycaemia includes either intramuscular glucagon or the intravenous administration of $10 \%$ glucose, in $100 \mathrm{ml}$ boluses to a maximum of $300 \mathrm{ml}(30 \mathrm{~g}){ }^{11}$ Similar guidelines to those used in the study by Holstein et $a l,{ }^{13}$ ie, continuous infusion of $10 \%$ glucose after the initial bolus, could be considered for UK ambulance clinicians. This may be particularly beneficial in remote and rural areas, should such a situation arise, where transportation times can be prolonged and repeat intervention may be required.

\section{Future directions: education and referral processes}

Some ambulance service guidelines currently enable clinicians to refer or discharge patients with certain conditions, including hypoglycaemia. However, although basic ambulance service education includes the recognition of and emergency treatments for patients with hypoglycaemia, there is little specific education on the risks associated with OHA or the provision of appropriate care plans (ie, what to do with patients). Lack of awareness of the possibility of a RHE may result in patients being left at home inappropriately by ambulance clinicians. The literature currently suggests a minimum observation period of $24 \mathrm{~h}$ for post-hypoglycaemic patients treated with OHA. Extended observation would be difficult in an outpatient setting, the literature did not suggest this as an alternative and it is unlikely that the current health service infrastructure would enable this to be achieved safely. Therefore, patients treated with OHA who have had hypoglycaemic events requiring treatment from an ambulance clinician should be transported directly to hospital so they can be observed, treated and receive appropriate follow-up care. Ambulance clinicians should be educated to this effect.

Although it is not always necessary to transport the posthypoglycaemic type 1 insulin-dependent patient with diabetes, this review has highlighted other issues that require to be addressed with this population. Some S\&T guidelines place the responsibility of contacting the general practitioner following a hypoglycaemic event on the patient; ${ }^{4}$ ambulance clinicians typically leave a carbon copy of the S\&T form containing details of the event and the patient is asked to pass this on to their general practitioner. This system is ineffective. Patients' attendance for follow-up in primary care (which enables medication alteration and specialist education to be provided) is low, ${ }^{17}$ and when patients do make an appointment the time delay between the original referral and attendance can often be several days, during which time a relapse may occur. ${ }^{6}$ Walker et $a l^{6}$ developed and evaluated a referral process for posthypoglycaemic patients. Patients who had had a hypoglycaemic episode were referred by the ambulance service to a diabetic specialist nurse (DSN) who then contacted the patient within 7 days of the event. Fifty-three per cent $(n=20)$ of the referred patients required medication alteration and $37 \%$ $(\mathrm{n}=14)$ required additional appointments for ongoing care issues. One patient had a further hypoglycaemic event in the period between being referred and being seen by the DSN.

Irrespective of the type of diabetes, efforts should be made to increase the attendance of these patients to primary or specialist care within as short a timeframe as possible. This would enable more patients to receive expert advice, medication review and subsequent follow-up care. Evidence-based interventions to increase post-hypoglycaemic patient attendance in primary care should be developed.

\section{LIMITATIONS}

Only English language publications were included in this review. Although we have comprehensively searched and appraised the available literature, little high quality research was retrieved. Consequently, caution is required in interpreting the findings of the studies.

\section{CONCLUSION}

The findings of this study indicate that post-hypoglycaemic patients treated in the prehospital environment are at a $2-7 \%$ risk of experiencing a RHE within $48 \mathrm{~h}$. The potential for OHA to cause RHE is highlighted in the reviewed literature. However, the extent to which this occurs in practice remains unknown. This lack of evidence has led to the recommendation that conservative management, through admission to hospital, is appropriate. The review has also highlighted that appropriate follow-up care of all other post-hypoglycaemic patients is required.

Several practice and research recommendations arise from this review.

Practice recommendations:

- All hypoglycaemia S\&T guidelines should recommend that patients treated with $\mathrm{OHA}$ are transported to hospital for appropriate observation and treatment. Ambulance clinicians should receive education on the appropriate management of hypoglycaemic patients treated with OHA.

- Referral pathways should be developed to ensure appropriate follow-up care for all patients with diabetes.

Research recommendations:

- Evidence-based interventions to increase post-hypoglycaemic patient attendance in primary care should be developed and evaluated.

- An investigation into the causes of RHE, the timeframes in which these occur and the length of observation time required to ensure patient safety.

Acknowledgements: The authors would like to thank Professor Bruce Guthrie, Mr William Mason, Mr Keith Colver, Mr Robin Lawrenson, Mr Richard Whitfield and Fiona Mair for their assistance and advice in the development of this paper. They would also like to express their gratitude to Dr RG Pirrallo and Dr J Steinmetz who provided additional information on their research.

Funding: This review was funded by the Scottish Ambulance Service/Nursing Midwifery and Allied Health Professions Research Unit.

Competing interests: None.

Ethics approval: None.

\section{REFERENCES}

1. Scottish Government. Scottish diabetes framework action plan. Edinburgh: Scottish Government, 2006.

2. Diabetes UK. Reports and statistics: diabetes prevalence. http://www.diabetes.org uk/Professionals/Information_resources/Reports/Diabetes-prevalence-2007/ (accessed: 19 Sept 2008).

3. Scottish Executive. A national framework for service change: building a service fit for the future. Edinburgh: Scottish Executive, 2005. 
4. Scottish Ambulance Service. Scottish Ambulance Service: treat and refer guidelines. Edinburgh: SAS, 2004.

5. Welsh Ambulance Service. Welsh Ambulance Service: treat and refer guidelines. Denbighshire: WAS, 2006

6. Walker A, James C, Bannister M, et al. Evaluation of a diabetes referral pathway for the management of hypoglycaemia following emergency contact with the ambulance service to a diabetes specialist nurse team. Emerg Med J 2006;23:449-51.

7. British National Formulary. BNF.6.1.2.1 sulphony/ureas. http://bnf.org/bnf/bnf/57/ 4164.htm? $q=\% 22$ sulphonylureas\%22\# hit/ (accessed: 19 Sept 2008).

8. Harrigan RA, Nathan MS, Beattie P. Oral agents for the treatment of type 2 diabetes mellitus: pharmacology, toxicity, and treatment. Ann Emerg Med 2001;38:68-78.

9. Spiller HA. Management of antidiabetic medications in overdose. Drug Safety 1998;19:411-24

10. BASICs Education Scotland/Scottish Ambulance Service. Basics Education Scotland: paramedic extended skills course. Aberuthven: BASICs Education Scotland/ SAS, 2004.

11. Joint Royal College Ambulance Liason Committee. UK Ambulance Service Clinical Practice Guidelines (2006): glycaemic emergencies in adults. London: JRCALC, 2006.

12. Mattila EM, Kuisma MJ, Sund KP, et al. Out-of-hospital hypoglycaemia is safely and cost-effectively treated by paramedics. Eur J Emerg Med 2004;11:70-4.

13. Holstein A, Plaschke A, Vogel MY, et al. Prehospital management of diabetic emergencies-a population-based intervention study. Acta Anaesthesiol Scand 2003:47:610-15

14. Anderson S, Hogskilde PD, Wetterslev J, et al. Appropriateness of leaving emergency medical service treated hypoglycemic patients at home: a retrospective study. Acta Anaesthesiol Scand 2002;46:464-8.

15. Leese GP, Wang J, Broomhall J. Frequency of severe hypoglycemia requiring emergency treatment in type 1 and type 2 diabetes: a population-based study of health service resource use. Diabetes Care 2003;26:1176-80.
16. Socransky SJ, Pirrallo RG, Rubin JM, et al. Out-of-hospital treatment of hypoglycemia: refusal of transport and patient outcome. Acad Emerg Med 1998:5:1080-5.

17. Cain ED, Ackroyd-Stolarz S, Alexiadis P, et al. Prehospital hypoglycemia: the safety of not transporting treated patients. Prehosp Emerg Care 2003:4:458-65

18. Steinmetz J, Nielsen SL, Rasmussen LS. Hypoglycaemia in patients with diabetes: do they prefer prehospital treatment or admission to hospital? Eur J Emerg Med 2006;13:319-20.

19. Carter AJ, Keane PS, Dreyer JF. Transport refusal by hypoglycemic patients after onscene intravenous dextrose. Acad Emerg Med 2002;9:855-7.

20. Brackenridge $\mathbf{A}$, Wallbank $\mathrm{H}$, Lawrenson RA, et al. Emergency management of diabetes and hypoglycaemia. Emerg Med J 2006;23:183-5.

21. Lheureux PE, Zahir S, Penaloza A, et al. Bench-to-bedside review: antidotal treatment of sulfonylurea-induced hypoglycaemia with octreotide. Crit Care 2005; 6:543-9.

22. Murphy P, Colwell C. Prehospital management of diabetes. Emerg Med Serv 2000;29:78-85.

23. Roberts K, Smith A. Outcome of diabetic patients treated in the prehospital arena after a hypoglycaemic episode, and an exploration of treat and release protocols: a review of the literature. Emerg Med J 2003;20:274-6.

24. Zammitt NN, Frier BM. Hypoglycemia in type 2 diabetes: pathophysiology, frequency, and effects of different treatment modalities. Diabetes Care 2005;28:2948-61.

25. Langford NJ, Krentz AJ, Martin U, et al. Severe relapsing sulphonylurea-induced hypoglycaemia: a diagnostic and therapeutic challenge. Postgrad Med J 2003; 79:120-3.

26. Bussing R, Gende A. Severe hypoglycemia from clarithromycin-sulfonylurea drug interaction. Diabetes Care 2002;25:1659. 